\title{
The child's immune system and pediatric tuberculosis
}

\author{
Joseph A Bellanti ${ }^{*}$, Henry Yeager ${ }^{2}$, Barbara Zeligs ${ }^{1}$, Stephen M Peters ${ }^{3}$, Shahla Riazi ${ }^{1}$, Onorina Di Mita', \\ German Benavides ${ }^{1}$, Benjamin Sablan $\mathrm{Jr}^{4}$, Natalie Quion ${ }^{5}$ \\ From Immunodiagnosis of Tuberculosis: New Questions, New Tools \\ Virginia, VA, USA. 21-23 September 2008
}

That children are more likely develop a severe form of TB is reflective of the differences in the maturational stages of their immune systems, but a paucity of data is available about how this system matures and what the relationship of these developmental immune deficiencies are with infection. Maturational deficiencies in the adaptive and innate immune systems in infants and young children may result in immature macrophage and DC function; Th1-type responses to pathogens; and a propensity to develop Th2-type CD4 T-cells in response to immunogens.

In vitro responses of two groups of TST positive children at risk for TB were examined by comparing Enzyme-Linked ImmunoSorbent Assay (ELISA)-based IGRAs with clinical and TST findings. Age-related changes in the immune capacity for specific and mitogen-induced IFN- $\gamma$ production was also examined in these two groups. In the original guidelines for the use of QFT-G, the US Centers for Disease Control and Prevention recommended that additional studies were needed, especially in children under five years of age, both to establish the validity of the assay as a diagnostic tool in the younger age group and to compare the accuracy of the test with the TST for diagnosing active and latent TB. Of TST positive US children, 10 of the 196 (5\%) were found to be QFT-G positive; nine had an indeterminate response; of the 130 children from the Philippines who had been immunized with BCG, 115 were TST positive, and seven were also QFT-G positive. In BCG immunized children, all were TST positive and BCG-immunized and because of a presumptive

\footnotetext{
* Correspondence: bellantj@georgetown.edu

'Department of Pediatrics and Microbiology-Immunology and the International Center for Interdisciplinary Studies of Immunology, Georgetown University Medical Center, Washington, DC, USA

Full list of author information is available at the end of the article
}

diagnosis of $\mathrm{TB}$, all had received anti-TB therapy; of the 30 children, 14 (47\%) were found to be QFT-G-IT positive and one had an indeterminate response. For the study of variations in age-related immune capacity, the capacity of IFN- $\gamma$ production was measured in various age groups of children in response to specific TB peptides (ESAT-6, CFP-10 and TB7.7) as well as to mitogen in amounts used in the QFT-G and the QFT-G-IT assay kits that had been provided. Both studies suggest that ELISA QFT-G and QFT-G-IT assays are useful for diagnosing TB in children and that adequate IFN- $\gamma$ production was observed in all children in both groups following lymphocyte stimulation by either purified TB peptides or mitogen including those less than 5 years of age.

\section{Acknowledgements \\ The study was supported in part by research grants from NIH RO3AI060856 and from the MedStar Research Institute FY2007IRGA-04. \\ Author details \\ ${ }^{1}$ Department of Pediatrics and Microbiology-Immunology and the International Center for Interdisciplinary Studies of Immunology, Georgetown University Medical Center, Washington, DC, USA. ${ }^{2}$ Department of Medicine, Georgetown University Medical Center, Washington, DC, USA. ${ }^{3}$ Department of Pathology, Georgetown University Medical Center, Washington, DC, USA. ${ }^{4}$ Department of Pediatrics, Philippine General Hospital, Phillipines. ${ }^{5}$ Children's National Medical Center, Washington, DC, USA.}

Published: 17 December 2010

doi:10.1186/1753-6561-4-S3-011

Cite this article as: Bellanti et al:: The child's immune system and pediatric tuberculosis. BMC Proceedings 2010 4(Suppl 3):O11. 